\title{
BMJ Open Hyperkalaemia prevalence, recurrence and treatment in patients on haemodialysis in China: protocol for a prospective multicentre cohort study (PRECEDE-K)
}

\author{
Zhaohui Ni, ${ }^{1}$ Haijiao Jin (1) , ${ }^{1}$ Renhua Lu (1) , ${ }^{1}$ Li Zuo, ${ }^{2}$ Weimin Yu, ${ }^{3}$ Yuqing Ren, ${ }^{4}$ \\ Qiongqiong Yang, ${ }^{5}$ Jie Xiao, ${ }^{6}$ Qinghong Zhang, ${ }^{7}$ Lihong Zhang, ${ }^{8}$ Xinzhou Zhang, ${ }^{9}$ \\ Qinkai Chen, ${ }^{10}$ Chaosheng Chen, ${ }^{11}$ Guojian Shao, ${ }^{12}$ Qun Luo, ${ }^{13} \mathrm{Li}$ Yao, ${ }^{14}$
} Shuguang Qin, ${ }^{15}$ Hui Peng, ${ }^{16}$ Qing Zhao, ${ }^{17}$ the PRECEDE-K Study group

To cite: Ni Z, Jin H, Lu R, et al. Hyperkalaemia prevalence, recurrence and treatment in patients on haemodialysis in China: protocol for a prospective multicentre cohort study (PRECEDE-K). BMJ Open 2021;11:e055770. doi:10.1136/ bmjopen-2021-055770

- Prepublication history and additional material for this paper are available online. To view these files, please visit the journal online (http://dx.doi. org/10.1136/bmjopen-2021055770).

ZN and $\mathrm{HJ}$ contributed equally.

Received 26 July 2021 Accepted 05 November 2021

Check for updates

(C) Author(s) (or their employer(s)) 2021. Re-use permitted under CC BY-NC. No commercial re-use. See rights and permissions. Published by BMJ.

For numbered affiliations see end of article.

Correspondence to

Dr Zhaohui Ni;

profnizh@126.com

\section{ABSTRACT}

Introduction Hyperkalaemia (HK) is a potentially lifethreatening electrolyte imbalance associated with several adverse clinical outcomes and is common in patients with kidney failure. However, there is no evidence on the occurrence, recurrence and treatment of $\mathrm{HK}$ in patients on haemodialysis (HD) in China.

Methods and analysis The HK Prevalence,

Recurrence, and Treatment in Haemodialysis Study is a prospective, multicentre, observational, cohort study being conducted across 15-18 sites in China. Approximately 600 patients with end-stage kidney disease on HD are anticipated to be enrolled and will be followed up for 24 weeks. Patients will be in the long interdialytic interval (LIDI) at enrolment and will receive follow-up care every 4 weeks in LIDI for pre-dialysis and post-dialysis (at enrolment only) serum potassium measurements. To obtain pre-dialysis serum potassium levels in the short interdialytic interval (SIDI), a followup visit will be performed in the SIDI during the first week. Information on concomitant medications, blood gas analysis and biochemistry measurements will be obtained at enrolment and at each follow-up visit. The primary endpoint will be the proportion of patients experiencing HK (defined as serum potassium level $>5.0 \mathrm{mmol} / \mathrm{L}$ ) at the study enrolment or during the 24week follow-up. The key secondary endpoint will be the proportion of patients experiencing HK recurrence (defined as any HK event after the first HK event) within 1-6 months (if applicable) during the 24-week followup, including enrolment assessment.

Ethics and dissemination This study has been approved by Shanghai Jiaotong University School of Medicine, Renji Hospital Ethics Committee (2020040). Other participating subcentres must also obtain ethics committee approval prior to the start of the study. The Good Clinical Practice regulations shall be strictly followed during the test implementation. Amendments to the protocol will be reviewed by the ethics committees. Written informed consent will be obtained from all participants before collection of any

\section{Strengths and limitations of this study}

- This will be the first prospective study on the prevalence, recurrence and treatment pattern of hyperkalaemia in patients on haemodialysis in China and will provide high-quality evidence and meaningful insights for guiding physicians in clinical practice.

- The study will provide data on the treatment patterns for different options for the management of hyperkalaemia in haemodialysis patients, but will not focus on the efficacy, safety and tolerability of different treatments.

- The study will not focus on the prognosis or the impact of hyperkalaemia on cardiovascular events.

patient data and patient information. The findings of this study will be disseminated through peer-reviewed publications and conference presentations.

Trial registration number ClinicalTrials.gov Registry (NCT04799067).

\section{INTRODUCTION}

The prevalence of end-stage renal disease (ESRD) is on the rise globally. In 2010, 2.618 million patients with ESRD received renal replacement therapy (RRT) worldwide and its use is projected to be more than double by 2030 , with the highest prevalence in Asia. ${ }^{1}$ Haemodialysis (HD) is the dominant treatment modality for those who are under RRT in China. According to the report of the Chinese National Renal Data System in 2018, there were $>1.3$ million patients with ESRD in China, among whom there were 580000 patients who were on HD.

Hyperkalaemia (HK), a potentially lifethreatening electrolyte imbalance, can be classified depending on the severity 
of serum potassium levels as mild $(5.1-5.9 \mathrm{mmol} / \mathrm{L})$, moderate $(6.0-6.9 \mathrm{mmol} / \mathrm{L})$ and severe $(\geq 7 \mathrm{mmol} / \mathrm{L}){ }^{2}$ Due to its detrimental effects on cardiac electrophysiology and neuromuscular function, HK has been proven to be associated with several adverse clinical outcomes, including significant arrhythmia, hospitalisation and associated length of stay, and all-cause mortality. ${ }^{3-5} \mathrm{HK}$ is common among patients with kidney failure due to diminished renal potassium excretion, with a prevalence of $30 \%-50 \%$ in patients with ESRD under maintenance HD (MHD) worldwide, and is recognised as a risk factor for sudden death and all-cause mortality in patients on HD. ${ }^{67}$ Chinese patients with ESRD have different spectrum of chronic kidney disease $(\mathrm{CKD}),{ }^{8}$ and different dietary style and treatment patterns, ${ }^{9}$ thus they may have distinct characteristics of HK occurrence and recurrence. Besides, abnormalities of potassium, rapid changes in potassium concentrations during the HD session, have been suggested as a potential cause of cardiac arrhythmia. ${ }^{10-13}$ High dietary $\mathrm{K}^{+}$intake has been associated with increased 5-year mortality rates in patients on HD. Therefore, dietary $\mathrm{K}^{+}$intake should be restricted to $2000 \mathrm{mg} /$ day and patients should be educated about dietary habits, and dietary regimens should be personalised for individual patients. Moreover, HK can be managed by changes in dialysis prescriptions like potassium dialysate $\left(\mathrm{K}^{+} \mathrm{D}\right)$ concentration and number of HD sessions. ${ }^{14} \mathrm{~A}$ higher incidence of pre-dialysis $\mathrm{HK}$ after the long interdialytic interval (LIDI) was observed with $\mathrm{K}^{+} \mathrm{D}(\leq 2 \mathrm{mmol} / \mathrm{L}$ vs $\geq 3 \mathrm{mmol} / \mathrm{L}$ ). Also, three times per week of HD is associated with excess volume and metabolic fluctuations during the LIDI predisposing to cardiovascular morbidity and mortality. ${ }^{14}$

However, there is no evidence on the occurrence, recurrence and treatment of $\mathrm{HK}$ in patients undergoing HD in China. Meanwhile, there are no Chinese guidelines on HK management in patients undergoing HD. In the present study, we aimed to evaluate the prevalence and recurrence of HK in Chinese patients on HD and to understand the treatment pattern of HK in China.

\section{METHODS AND ANALYSIS \\ Design}

This is a prospective, observational, cohort study, which was registered with the ClinicalTrials.gov prior to initiation of patient enrolment.

Patients will be in LIDI at enrolment visit 1 (V1). Demographic characteristics, medical history, aetiology of ESRD, concomitant medications, dialysis vintage, concentration of $\mathrm{K}^{+} \mathrm{D}(0,1,2,3 \mathrm{mmol} / \mathrm{L}$ or other concentration), an ECG, a pre-dialysis $\mathrm{K}^{+}$measurement and a post-dialysis $\mathrm{K}^{+}$measurement, complete blood count, blood gas analysis and other blood biochemistry measurements will be obtained. Potassium measurements, including pre-dialysis potassium measurements at the LIDI once every month (V3-V8), and pre-dialysis potassium at short interdialytic interval (SIDI) during the first week (V2) for patients in HD, three times per week specifically, will be performed. Information on concomitant medications, complete blood count, blood gas analysis and other blood biochemistry measurements will also be obtained. Besides that, evaluation of the different ions $\left(\mathrm{K}^{+}, \mathrm{Na}, \mathrm{Mg}, \mathrm{Ca}\right.$ and $\left.\mathrm{P}\right)$ on a monthly basis, during the follow-up time with the different potassium chelators in patients on HD, will be conducted. In this study, control on dietary intake of potassium and dialysis prescription management will be carried out for the control of $\mathrm{HK}$ in addition to the therapy.

LIDI is defined as the interval between dialysis $\geq 2$ days, while SIDI is defined as the 1-day interval between dialysis. For patients receiving HD three times per week, there will be one LIDI and two SIDIs every week. For patients receiving HD two times per week, there will be two LIDIs every week.

The illustration of the proposed study design is shown in figure 1 (patient on HD three times per week as an example). The detailed study plan of the HK Prevalence, Recurrence, and Treatment in Haemodialysis (PRECEDE-K) Study is shown in table 1.

\section{Study sites and period}

This study will use primary data collected by investigators from approximately 15-18 sites in China. Patients

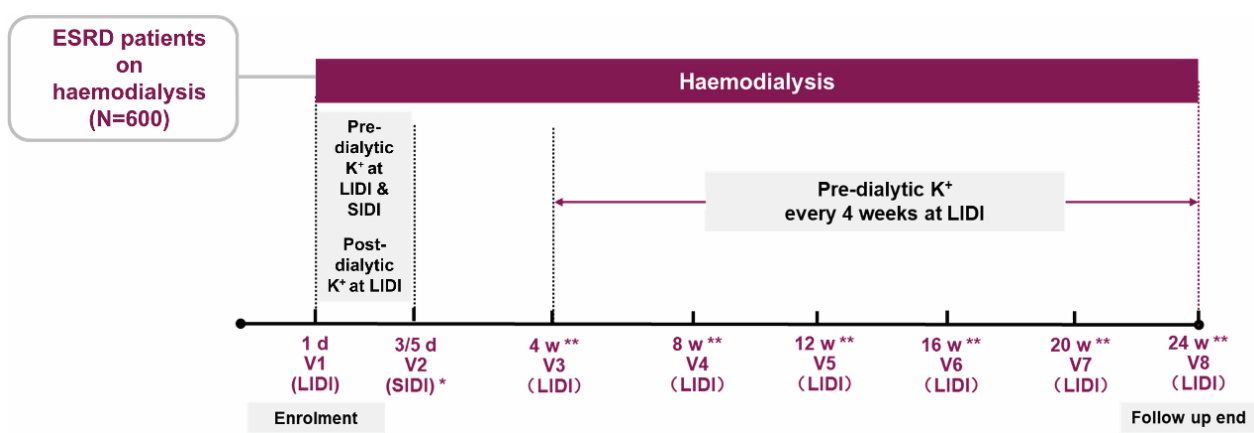

Figure 1 The study design of the PRECEDE-K Study. *For patients receiving HD three times per week, V2 could be at d3 or d5 for collecting pre-dialytic serum potassium at SIDI. For patients receiving HD two times per week, visit 2 will be waived as there is no SIDI for these patients; thus, there are seven visits in total. ${ }^{*}$ Time window is \pm 1 week. d, day; ESRD, end-stage renal disease; LIDI, long interdialytic interval; PRECEDE-K, HK Prevalence, Recurrence, and Treatment in Haemodialysis; SIDI, short interdialytic interval; V, visit; w, week. 
Table 1 The Study Plan of the PRECEDE-K Study

\begin{tabular}{|c|c|c|c|}
\hline & Visit 1 & Visit 2 & Visit 3-8 \\
\hline & 1 day (LIDI) & $3 / 5$ days ${ }^{*}$ (SIDI) & 4-24 weeks† (LIDI) \\
\hline ICF & $\mathrm{X}$ & & \\
\hline Screen inclusion and exclusion criteria & $\mathrm{X}$ & & \\
\hline Demographic characteristics & $\mathrm{X}$ & & \\
\hline Medical history & $\mathrm{X}$ & & \\
\hline Aetiology of ESRD & $\mathrm{X}$ & & \\
\hline Vascular access & $\mathrm{X}$ & & $\mathrm{X}$ \\
\hline Height $(\mathrm{cm})$ & $\mathrm{X}$ & & \\
\hline Pre-dialysis weight (kg) & $\mathrm{X}$ & $\mathrm{X}$ & $\mathrm{X}$ \\
\hline Post-dialysis weight (kg) & $\mathrm{X}$ & $\mathrm{X}$ & $\mathrm{X}$ \\
\hline Vital signs & $\mathrm{X}$ & $\mathrm{X}$ & $\mathrm{X}$ \\
\hline Physical examination & $\mathrm{X}$ & $\mathrm{X}$ & $\mathrm{X}$ \\
\hline Pre-dialysis serum $\mathrm{K}^{+} \ddagger$ (mEq/L) & $\mathrm{X}$ & $\mathrm{X}$ & $\mathrm{X}$ \\
\hline Post-dialysis serum $\mathrm{K}^{+} \ddagger$ (mEq/L) & $\mathrm{X}$ & & $\mathrm{X}^{*}$ \\
\hline Dialysis adequacy§ & $\mathrm{X}$ & & Evaluated at week 12 \\
\hline Dialysis frequency & $\mathrm{X}$ & & $\mathrm{X}$ \\
\hline Dialysis prescription & $\mathrm{X}$ & & $\mathrm{X}$ \\
\hline ECGף & $\mathrm{X}$ & & $\mathrm{X}^{*}$ \\
\hline Echocardiography ${ }^{\star \star}$ & $X^{\star}$ & & $\mathrm{X}^{\star}$ \\
\hline Urine 24-hour volume (L) & & & Evaluated at week 4 or week 8 \\
\hline Urine biochemistry measurements $\dagger \dagger$ & & & Evaluated at week 4 or week 8 \\
\hline Ultra-filtration rate & $\mathrm{X}$ & & $\mathrm{X}$ \\
\hline Complete blood count & $\mathrm{X}$ & & $\mathrm{X}$ \\
\hline Biochemistry measurements & $\mathrm{X}$ & & $\mathrm{X}$ \\
\hline Blood gas analysis & $\mathrm{X}$ & & $\mathrm{X}$ \\
\hline Concomitant medication & $\mathrm{X}$ & $\mathrm{X}$ & $\mathrm{X}$ \\
\hline
\end{tabular}

Vital signs, physical examination, pre-dialysis $\mathrm{K}^{+}$, pre-dialysis weight, complete blood count and biochemistry measurements should be collected within 30 min before initiation of HD. Post-dialysis weight, post-dialysis $\mathrm{K}^{+}$and post-dialysis BUN should be collected within 30 min after HD procedure.

All blood samples will be measured at a local laboratory.

*Not study defined specific mandatory assessments. Records will only be collected if available during standard usual clinical practice

${ }^{*}$ For patients receiving HD three times per week, there are two SIDIs in an HD session, so visit 2 can be on day 3 or day 5 . For patients receiving HD two times per week, visit 2 will be waived, as there is no SIDI for these patients.

†Time window is \pm 1 week.

$\ddagger$ ‡lood samples for the determination of pre-dialysis serum $\mathrm{K}^{+}$concentration and post-dialysis serum $\mathrm{K}^{+}$concentration will be drawn before and after the HD procedure according to the routine clinical practice.

$\S$ Dialysis adequacy will be evaluated by URR. URR $=\left(C_{0}-C\right) / C_{0} ; C_{0}$ : pre-dialysis BUN, C: post-dialysis $\left.B U N\right)$. To calculate the

URR, post-dialysis BUN will be tested on day 1 and week 12 .

IECG: evaluated within 1 day before or after the enrolment in principle; allow individual adjustments in each centre. All ECGs during the follow-up should be recorded in eCRF. For patients who experience HK during the study period, it is recommended to conduct at least one more pre-dialysis ECG at LIDI.

${ }^{* *}$ Echocardiography: evaluated echocardiography data if it is conducted within 2 weeks before or after visits in principle; allow individual adjustments in each centre.

††Urine will be collected for a period of 24 hours at either week 4 or week 8 . The volume and biochemistry measurements of the 24-hour urine sample will be measured.

BUN, Blood urea nitrogen; eCRF, electronic case report form; ESRD, end-stage renal disease; HD, haemodialysis; HK, hyperkalaemia; ICF, intracellular fluid; LIDI, long interdialytic interval; PRECEDE-K, HK Prevalence, Recurrence, and Treatment in Haemodialysis; SIDI, short interdialytic interval; URR, Urea Reduction Ratio. 
with ESRD on HD treatment will be enrolled and will be followed up for 24 weeks.

\section{Population}

This study will include approximately 600 patients with ESRD (aged $\geq 18$ years) receiving HD treatment two times per week or three times per week. Written informed consent will be obtained from all the participants (online supplemental file 1). Patients will be eligible to be included in the study only if all of the following criteria are applicable: (1) patients are aged $\geq 18$ years at the time of signing the informed consent; (2) patients with ESRD and on HD; (3) the HD treatment frequency is $\geq 2$ sessions per week; (4) capable of giving signed informed consent.

Patients will be excluded from the study if any of the following criteria are applicable: (1) acute kidney injury; (2) expected to receive renal transplantation within 6 months; (3) intracranial haemorrhage or elevated intracranial pressure within 1 month before enrolment; (4) a traumatic experience that cannot be corrected by drugs within 1 month before enrolment; (5) failure to establish vascular access; (6) has been receiving peritoneal dialysis; (7) not suitable for this study as judged by the investigators.

\section{Procedures for withdrawal/discontinuation}

Eligible patients will be followed for up to 24 weeks, or up until occurrence of any of the following events, whichever occurred earlier.

1. Death.

2. End of study period.

3. Loss to follow-up: defined as transfer to HD centre(s) not in this study, or refused to continue follow-up, or cannot be contacted by phone calls for three times.

4. Change modality of RRT (peritoneal dialysis or renal transplantation).

5. Withdrawal of intracellular fluid (ICF).

6. Investigators consider it necessary for the subjects to terminate the study.

\section{Primary outcome}

The primary outcome will be proportion of patients experiencing HK (defined as serum potassium levels $>5.0 \mathrm{mmol} / \mathrm{L}$ ) at the study enrolment or during a 24-week follow-up.

\section{Secondary outcomes}

- The proportion of patients experiencing HK recurrence (defined as any HK event after the first HK event) within 1, 2, 3, 4, 5 or 6 months (if applicable) during a 24-week follow-up including the enrolment assessment. An HK event is defined as any serum $\mathrm{K}^{+}$ concentration $>5.0 \mathrm{mmol} / \mathrm{L}$ within an interdialytic interval, which is usually $2-3$ days.

- The proportion of patients with 2, 3, 4, 5 and $\geq 6$ events of HK during a 24-week follow-up including the enrolment assessment.
- Intradialytic potassium shift (defined as the difference between pre-dialysis and post-dialysis $\mathrm{K}^{+}$concentration) at LIDI during the first week after patient enrolment.

- Serum $\mathrm{K}^{+}$concentration at LIDI and SIDI in patients receiving HD three times per week during the first week after patient enrolment.

- The proportion of patients with HK treated with any potassium binders including sodium polystyrene sulfonate (SPS), calcium polystyrene sulfonate (CPS), or sodium zirconium cyclosilicate (SZC), and specific proportion of each potassium binder, respectively, during the 24-week follow-up period.

- The proportion of HK events treated with any potassium binders including SPS, CPS or SZC among total number of HK events during the 24-week follow-up period.

- Mean daily dose of SPS, CPS or SZC in patients treated with any potassium binder.

- Duration of the treatment of SPS, CPS or SZC in patients treated with any potassium binders.

\section{Exploratory endpoints}

- Risk factors for experiencing any HK (defined as serum potassium level $>5.0 \mathrm{mmol} / \mathrm{L}$ ) at the study enrolment or during a 24-week follow-up.

- Risk factors for experiencing HK recurrence during a 24-week follow-up.

- The efficacy, safety and tolerability of the options for the management of HK in HD patients.

\section{Statistical analysis principles}

Statistical methods will be primarily descriptive in nature. For categorical data, the frequency and percentage of patients in each category will be presented. Percentages will be based on non-missing data unless otherwise specified. For continuous data, descriptive statistics will be presented as the number of patients (n), mean, $\mathrm{SD}$, median, minimum and maximum. All successfully enrolled subjects fulfilling inclusion/exclusion criteria will be included in the Full Analysis Set (FAS), which will be the primary analysis set for all primary and secondary analyses unless specified otherwise.

\section{Primary analysis}

The proportion of patients experiencing any HK at the study enrolment or during a 24-week follow-up will be presented by the percentage as well as its 95\% CI. HK is defined as $\mathrm{K}^{+}>5.0 \mathrm{mmol} / \mathrm{L}$, while the same summary will be generated using a higher threshold of $\mathrm{HK}$ as $\mathrm{K}^{+}$ $>5.5 \mathrm{mmol} / \mathrm{L}$, as well as sensitivity analysis. The proportion of patients experiencing serum $\mathrm{K}^{+}$concentration $>6.0 \mathrm{mmol} / \mathrm{L}, 6.5 \mathrm{mmol} / \mathrm{L}, 7.0 \mathrm{mmol} / \mathrm{L}$ and $7.5 \mathrm{mmol} / \mathrm{L}$ will be reported as well.

\section{Secondary analysis}

1. The proportion of HK recurrence within 1, 2, 3, 4, 5 or 6 month (s) after the first HK and the proportions of patients with $2,3,4,5$ and $\geq 6$ times of HK events during 
the 24-week follow-up will be presented using the percentage and its $95 \%$ CI. The HK recurrence is defined as any consecutive HK events. An HK event is defined as any serum $\mathrm{K}^{+}$concentration $>5.0 \mathrm{mmol} / \mathrm{L}$ within an interdialytic interval, which is usually $2-3$ days. A higher threshold of HK event as serum $\mathrm{K}^{+}$concentration $>5.5 \mathrm{mmol} / \mathrm{L}$ will be used to generate similar summaries, as sensitivity analyses.

2. Intradialytic potassium shift is evaluated by the mean difference between pre-dialysis and post-dialysis serum $\mathrm{K}^{+}$concentration during the first week after patient enrolment. If there is more than one serum $\mathrm{K}^{+}$concentration before or after HD, intradialytic potassium shift will be the difference between the last serum $\mathrm{K}^{+}$ concentration measurement before HD and the first serum $\mathrm{K}^{+}$measurement after HD. Summary statistics will be provided using mean, median, SD, minimum and maximum. The number of subjects and the missing number of subjects will also be presented.

3. Serum $\mathrm{K}^{+}$concentration at LIDI and SIDI as well as the difference between LIDI and SIDI during the first week after patient enrolment will be summarised with the same method as described above. This analysis will only be applicable to patients receiving HD three times per week. LIDI is defined as the interval between dialysis ( $\geq 2$ days), whereas SIDI is defined as the 1-day interval between dialysis.

4. Treatment pattern for HK with potassium binders will be assessed by: the proportion of patients with HK treated with any potassium binders including SPS or CPS or SZC during the 24-week follow-up period. The proportion of patients with HK treated with specific potassium binder including SPS, CPS and SZC, respectively, during the 24-week follow-up period; the proportion of HK events treated with any potassium binders including SPS, CPS or SZC among total number of HK events during the 24-week follow-up period; the mean daily dose of SPS, CPS or SZC in patients treated with any potassium binder; and the duration of the treatment of SPS, CPS or SZC in patients treated with any potassium binder, above the categorical and continuous variables, will be analysed based on previously mentioned descriptive methods, as associated summary statistics will be presented. Whereas for proportion, the denominator will always be the number of subjects in FAS.

\section{Exploratory analysis}

Univariate and multivariate logistic regressions will be used to explore the association of risk factors with any HK occurrence and recurrence. HK occurrence is defined similar to that mentioned in the primary analysis, where patients experience any HK (defined as serum potassium $>5.0 \mathrm{mmol} / \mathrm{L}$ ) at the study enrolment or during a 24-week follow-up. The HK recurrence is defined similar to that mentioned in the secondary analysis, where patients experienced any HK event after the first HK event will be counted as with recurrence. An HK event
Table 2 Precision estimates for primary endpoint under different sample sizes

\begin{tabular}{llll}
\hline HK proportion & Sample size & Precision & $95 \%$ Cl estimate \\
\hline $58.0 \%$ & 600 & $3.90 \%$ & $54.1 \%$ to $61.9 \%$ \\
$73.8 \%$ & 600 & $3.50 \%$ & $70.3 \%$ to $77.3 \%$ \\
\hline
\end{tabular}

HK, hyperkalaemia.

is defined as any serum $\mathrm{K}^{+}$concentration $>5.0 \mathrm{mmol} / \mathrm{L}$ within an interdialytic interval, which is usually $2-3$ days. Risk factors include dialysis frequency; $\mathrm{K}^{+} \mathrm{D}$; dialysis vintage; treatment of $\mathrm{HK}$; and medical history of special interest, including history of atherosclerotic heart disease, congestive heart failure, diabetes, hypertension, uncontrolled asthma on bronchodilators, dialysis adequacy, Urea reduction ratio (URR), urine volume and so on. OR and $95 \%$ CI estimation of each risk factor (both unadjusted and adjusted) will be presented with a two-sided $\mathrm{p}$ value provided. An exploratory subgroup analysis will be set to evaluate the efficacy, safety and tolerability of the options for the management of HK in HD patients.

\section{Sample size and power calculations}

The primary endpoint will be the proportion of patients experiencing HK anytime at enrolment and during a 24-week follow-up; the previously reported proportion of patients experiencing $\mathrm{HK}$ is $73.8 \%$ during a 2-year follow-up ${ }^{9}$ and $58 \%$ during a 4-month follow-up. ${ }^{11}$ In this study, an assumption is made that the proportion of patients experiencing HK will be between $58 \%$ and $73.8 \%$ during a 24-week follow-up. Precision estimates for primary endpoint under different sample sizes are presented in table 2. We assumed that 600 patients will provide a precision (half width of $95 \%$ CI) estimate of $3.5 \%-3.9 \%$.

\section{Data statement}

All data will be collected and entered into the electronic case report form (eCRF). The investigator will be responsible for ensuring that the required data are collected and entered into the eCRF.

\section{Quality control}

The activities for quality control could include but are not limited to:

\section{Contacts with the sites to:}

- Provide information and support to the investigator(s).

- Confirm that the research team is complying with the protocol and that data are being accurately recorded in the eCRFs.

- Ensure that the subject ICFs are signed and stored at the investigator's site.

- Ensure that the eCRFs are completed properly and with adequate quality.

Monitoring activities for:

- Checking of ICFs.

- Checking that subjects exist in medical records. 
The extent and nature of monitoring will be decided during the study planning based on design, complexity, number of subjects, number of sites, etc.

Different signals (eg, high rejection rate in a site) should be used as potential identification of low protocol compliance by investigators.

If these or any other signal occurs or if the investigator is suspicious of a potential non-optimal level of protocol compliance by the site investigator, specific measures should be adopted to evaluate the situation, identify the issue and implement specific action plans to correct the situation.

\section{Training of study site personnel}

The principal investigator will ensure that appropriate training relevant to the observational study is given to investigational staff.

\section{Patient and public involvement}

No patient involved.

\section{ETHICS AND DISSEMINATION}

This study has been approved by Shanghai Jiaotong University School of Medicine, Renji Hospital Ethics Committee (2020-040). Other participating subcentres must also obtain ethics committee approval documents prior to the start of the study. The Good Clinical Practice regulations shall be strictly followed during the test implementation. Amendments to the protocol will be reviewed by the ethics committees. Written informed consent will be obtained from all participants before collection of any patient data or patient information. The findings of this study will be disseminated through peer-reviewed publications and conference presentations.

\section{DISCUSSION}

HK is common among patients with kidney failure due to diminished renal potassium excretion despite the widespread and dynamic prescription of low- $\mathrm{K}^{+}$dialysis baths and $\mathrm{K}^{+}$binders. According to a prospective multicentre study in France, the percentages of patients with long-term HD experiencing $\mathrm{HK}$ at any time were $73.8 \%\left(\mathrm{~K}^{+}>5.1 \mathrm{mmol} / \mathrm{L}\right), 57.9 \%\left(\mathrm{~K}^{+}>5.5 \mathrm{mmol} / \mathrm{L}\right)$ and $34.5 \%\left(\mathrm{~K}^{+}>6 \mathrm{mmol} / \mathrm{L}\right) .{ }^{15}$ Another large retrospective study that analysed data derived from the US Renal Data System reported that HK prevalence was consistently estimated at $16.3-16.8$ events per 100 patient-months. ${ }^{16}$

Recently, an epidemiological study reported that the prevalence of HK in Chinese outpatients is $3.86 \%$, and the prevalence of $\mathrm{HK}$ in patients with CKD increased to $22.89 \% .{ }^{17}$ However, there is no high-quality evidence on the epidemiology of HK and no Chinese guidelines on the management of HK in Chinese patients on HD. The PRECEDE-K Study will evaluate the prevalence of HK in Chinese patients on HD.
Previously, acute HK was considered as a fatal complication; however, with the deepened understanding of $\mathrm{HK}$, emphasis has changed from acute HK to chronic HK in the recent years. Acute HK management involves cardiac monitoring, acute medical interventions or possibly dialysis. However, chronic HK requires ongoing management to correct the underlying disturbances in potassium balance including non-pharmacological and pharmacological interventions. ${ }^{18} \mathrm{~A}$ higher rate of HK recurrence was observed 1, 2 and 3 months after an HK occurrence (eg, $35.6 \%$ of $\mathrm{HK}$ cases with $\mathrm{K}^{+}>6 \mathrm{mmol} / \mathrm{L}$ within 3 months after an initial HK of the same magnitude) in hepatic haemosiderosis in MHD patients despite widespread prescription of low- $\mathrm{K}^{+}$dialysis baths and $\mathrm{K}^{+}$binders. ${ }^{15}$ Therefore, the proportion of patients experiencing HK recurrence is an important secondary outcome in this current study.

Compared with pre-dialysis $\mathrm{K}^{+}$, post-dialysis $\mathrm{K}^{+}$is rarely investigated, and so its acceptable range remains unknown. Based on the difference between dialysate and serum potassium levels, serum potassium levels drop significantly after $\mathrm{HD}$, and $45 \%$ of patients present with post-dialysis hypokalaemia of $<3.5 \mathrm{mmo} / \mathrm{L}$ in previous study. ${ }^{19-21}$ Based on a cohort study of 3967 participants on MHD from the Dialysis Outcomes and Practice Patterns Study in Japan (2009-2012 and 2012-2015) compared with post-dialysis potassium levels $3.0-<3.5 \mathrm{mEq} / \mathrm{L}$, the HRs of post-dialysis hypokalaemia $(<3.0 \mathrm{mEq} / \mathrm{L})$ were 1.84 (1.44 to 2.34$)$ in the unadjusted model, 1.44 (1.14 to 1.82 ) in the model without adjusting for pre-dialysis serum potassium levels, and 1.10 (0.84 to 1.44) in the model adjusted for pre-dialysis serum potassium levels. This research suggested that post-dialysis hypokalaemia was associated with mortality, but this association was not independent of pre-dialysis potassium. ${ }^{22}$ Therefore, we regarded intradialytic potassium shift (defined as the difference between pre-dialysis and post-dialysis $\mathrm{K}^{+}$) as a secondary outcome in our research.

A retrospective study reported that the prevalence of HK on the day after the LIDI was 2.0-2.4 times as high as on the day after the SIDI in patients with MHD. ${ }^{16}$ The LIDI was reported as a time of heightened risk among patients receiving $\mathrm{HD} .{ }^{23}$ Serum $\mathrm{K}^{+}$level at LIDI and SIDI in patients receiving HD three times per week during the first week after patient enrolment was another secondary outcome.

The development of HK is usually the result of a combination of factors superimposed on comorbidities (eg, diabetes mellitus, advanced stages of heart failure), use of potassium-based salt substitutes, and use of medications interfering with potassium homeostasis-like ACE inhibitors, angiotensin receptor blockers, aldosterone receptor antagonists, $\beta$-blockers and others. ${ }^{24}$ Considering the potential risk factor of HK, we will record all the comorbidities and drugs in this study for further exploration.

Compared with the reference group of serum potassium level of $4.0-5.0 \mathrm{mEq} / \mathrm{L}$, higher serum potassium level $(5.6-6.0 \mathrm{mEq} / \mathrm{L})$ was associated with mortality in 
adjusted analysis (HR: 1.13, 95\% CI: 1.06 to 1.20 ), and higher serum potassium level $(>6.0 \mathrm{mEq} / \mathrm{L})$ was associated with arrhythmia composite (includes sudden death or arrhythmia-related hospitalisations) in adjusted analysis (HR: $1.21,1.05$ to 1.38$).{ }^{4}$ Continuous rhythm monitoring was performed using the remote-monitoring capability of the implantable loop recorder device in patients undergoing $\mathrm{HD}$ at eight centres. ${ }^{25}$ In multivariate survival frailty analyses, a higher risk of conduction disorder was associated with plasma potassium levels $>5.0 \mathrm{mmol} / \mathrm{L}$. Further understanding and management of $\mathrm{HK}$ will benefit the survival in MHD patients.

However, while our study will provide data on the treatment patterns for different options for the management of HK in HD patients, it will not focus on the efficacy, safety and tolerability of different treatments. Additionally, the study will not focus on the prognosis or the impact of HK on cardiovascular events.

\section{Author affiliations}

${ }^{1}$ Department of Nephrology, Ren Ji Hospital, Shanghai Jiao Tong University School of Medicine, Shanghai, China

${ }^{2}$ Department of Nephrology, Peking University People's Hospital, Beijing, China

${ }^{3}$ Department of Nephrology, Shanxi Bethune Hospital, Taiyuan, China

${ }^{4}$ Department of Nephrology, Yangquan Coal Industry (Group) General Hospital,

Yangquan, China

${ }^{5}$ Department of Nephrology, Sun Yat-Sen Memorial Hospital, Sun Yat-Sen University, Guangzhou, Guangdong, China

${ }^{6}$ Department of Nephrology, The First Affiliated Hospital of Guangzhou Medical University, Guangzhou, Guangdong, China

${ }^{7}$ Department of Nephrology, Taihe Hospital, Shiyan, Hubei, China

${ }^{8}$ Department of Nephrology, The First Hospital of Hebei Medical University, Shijiazhuang, Hebei, China

${ }^{9}$ Department of Nephrology, Shenzhen People's Hospital, Shenzhen, Guangdong, China

${ }^{10}$ Department of Nephrology, First Affiliated Hospital of Nanchang University,

Nanchang, Jiangxi, China

${ }^{11}$ Department of Nephrology, The First Affiliated Hospital of Wenzhou Medical

University, Wenzhou, Zhejiang, China

${ }^{12}$ Department of Nephrology, Wenzhou Central Hospital, Wenzhou, Zhejiang, China

${ }^{13}$ Department of Nephrology, Hwa Mei Hospital, University of Chinese Academy of

Sciences, Ningbo, Zhejiang, China

${ }^{14}$ Department of Nephrology, The first hospital of China Medical University, Shenyang, China

${ }^{15}$ Department of Nephrology, Guangzhou First People's Hospital, Guangzhou, Guangdong, China

${ }^{16}$ Department of Nephrology, Third Affiliated Hospital of Sun Yat-Sen University,

Guangzhou, Guangdong, China

${ }^{17}$ Medical Affaires, AstraZeneca Investment China Co, Shanghai, China

Collaborators Haifeng Liu, Yifan Shi, Yan Wang, Xiaojun Chen, Chunyi Zhang, Yan Huang, Miao Xu, Wenlong Shao

Contributors Principal investigator (PI) and conception-ZN. Sub-PI and writingHJ. Sub-PI-RL. Acquisition of data and executive-LZ, WY, YR, QY, JX, QZ, LZ, XZ, $Q C, C C, G S, Q L, L Y, S Q$ and HP. Conception and design-QZ.

Funding The PRECEDE-K Study was supported by AstraZeneca Investment China $\mathrm{Co}$, Shanghai, China. This study was also funded by the Clinical Research Plan of SHDC (No. SHDC2020CR3029B) and the Multicenter Clinical Research Project of Shanghai Jiao Tong University School of Medicine (DLY201805).

Competing interests None declared.

Patient consent for publication Not required.

Provenance and peer review Not commissioned; externally peer reviewed.
Supplemental material This content has been supplied by the author(s). It has not been vetted by BMJ Publishing Group Limited (BMJ) and may not have been peer-reviewed. Any opinions or recommendations discussed are solely those of the author(s) and are not endorsed by BMJ. BMJ disclaims all liability and responsibility arising from any reliance placed on the content. Where the content includes any translated material, BMJ does not warrant the accuracy and reliability of the translations (including but not limited to local regulations, clinical guidelines, terminology, drug names and drug dosages), and is not responsible for any error and/or omissions arising from translation and adaptation or otherwise.

Open access This is an open access article distributed in accordance with the Creative Commons Attribution Non Commercial (CC BY-NC 4.0) license, which permits others to distribute, remix, adapt, build upon this work non-commercially, and license their derivative works on different terms, provided the original work is properly cited, appropriate credit is given, any changes made indicated, and the use is non-commercial. See: http://creativecommons.org/licenses/by-nc/4.0/.

\section{ORCID iDs}

Haijiao Jin http://orcid.org/0000-0003-2804-4702

Renhua Lu http://orcid.org/0000-0003-3555-505X

\section{REFERENCES}

1 Liyanage T, Ninomiya T, Jha V, et al. Worldwide access to treatment for end-stage kidney disease: a systematic review. Lancet 2015;385:1975-82.

2 Palmer BF, Carrero JJ, Clegg DJ, et al. Clinical management of hyperkalemia. Mayo Clin Proc 2021;96:744-62.

3 Nilsson E, Gasparini A, Ärnlöv J, et al. Incidence and determinants of hyperkalemia and hypokalemia in a large healthcare system. Int $J$ Cardiol 2017;245:277-84.

4 Karaboyas A, Zee J, Brunelli SM, et al. Dialysate potassium, serum potassium, mortality, and arrhythmia events in hemodialysis: results from the dialysis outcomes and practice patterns study (DOPPS). Am J Kidney Dis 2017:69:266-77.

5 Palaka E, Grandy S, Darlington O, et al. Associations between serum potassium and adverse clinical outcomes: a systematic literature review. Int J Clin Pract 2020;74:e13421.

6 Herzog CA, Mangrum JM, Passman R. Non-Coronary heart disease in dialysis patients: sudden cardiac death and dialysis patients: sudden cardiac death in dialysis patients. Semin Dial 2008;21:300-7.

7 Kovesdy CP, Regidor DL, Mehrotra R, et al. Serum and dialysate potassium concentrations and survival in hemodialysis patients. Clin J Am Soc Nephrol 2007;2:999-1007.

8 Huang Y-M, Xu D, Long J, et al. Spectrum of chronic kidney disease in China: a national study based on hospitalized patients from 2010 to 2015. Nephrology 2019;24:725-36.

9 Yan Y, Ramirez S, Anand S, et al. Twice-Weekly hemodialysis in China: can it be a better option for initiation or maintenance dialysis therapy? Semin Dial 2017;30:277-81.

10 Bleyer AJ, Hartman J, Brannon PC, et al. Characteristics of sudden death in hemodialysis patients. Kidney Int 2006;69:2268-73.

11 Genovesi S, Valsecchi MG, Rossi E, et al. Sudden death and associated factors in a historical cohort of chronic haemodialysis patients. Nephrol Dial Transplant 2009;24:2529-36.

12 Hung AM, Hakim RM. Dialysate and serum potassium in hemodialysis. Am J Kidney Dis 2015;66:125-32.

13 Labriola L, Jadoul M. Sailing between Scylla and Charybdis: the high serum K-low dialysate K quandary. Semin Dial 2014;27:463-71.

14 Bansal S, Pergola PE. Current management of hyperkalemia in patients on dialysis. Kidney Int Rep 2020;5:779-89.

15 Rossignol P, Lamiral Z, Frimat L, et al. Hyperkalaemia prevalence, recurrence and management in chronic haemodialysis: a prospective multicentre French regional registry 2-year survey. Nephrol Dial Transplant 2017;32:2112-8.

16 Yusuf AA, Hu Y, Singh B, et al. Serum potassium levels and mortality in hemodialysis patients: a retrospective cohort study. Am J Nephrol 2016:44:179-86.

17 Bian J, Zuo L, Zhao H, et al. P0799EPIDEMIOLOGY and treatment pattern of hyperkalaemia among outpatients in China: a descriptive study using an administrative database in China. Nephrol Dial Transplant 2020;35.

18 02-10-7259_DBH_Best-Practices-in-Managing-Hyperkalemia-inCKD.pdf. Available: https://www.kidney.org/sites/default/files/02-107259_DBH_Best-Practices-in-Managing-Hyperkalemia-in-CKD.pdf [Accessed 29 Sep 2021].

19 Nakai S, Hanafusa N, Masakane I. An overview of regular dialysis treatment in Japan (as of 31 December 2012): overview of dialysis treatment in Japan 2012. Ther Apher Dial 2014:535-602. 
20 Blumberg A, Roser HW, Zehnder C, et al. Plasma potassium in patients with terminal renal failure during and after haemodialysis; relationship with dialytic potassium removal and total body potassium. Nephrol Dial Transplant 1997;12:1629-34.

21 Agar BU, Culleton BF, Fluck R. Potassium kinetics during hemodialysis: hemodialysis potassium kinetics. Hemodial Int 2015;19:23-32.

22 Ohnishi T, Kimachi M, Fukuma S, et al. Postdialysis hypokalemia and all-cause mortality in patients undergoing maintenance hemodialysis. Clin J Am Soc Nephrol 2019;14:873-81.
23 Foley RN, Gilbertson DT, Murray T, et al. Long interdialytic interval and mortality among patients receiving hemodialysis. $N$ Engl J Med 2011;365:1099-107.

24 Sarafidis PA, Blacklock R, Wood E, et al. Prevalence and factors associated with hyperkalemia in predialysis patients followed in a low-clearance clinic. Clin J Am Soc Nephrol 2012;7:1234-41.

25 Sacher F, Jesel L, Borni-Duval C, et al. Cardiac rhythm disturbances in hemodialysis patients. JACC: Clinical Electrophysiology 2018;4:397-408. 